\title{
UNA NUEVA ESPECIE DE ELAPHOGLOSSUM SECCIÓN ELAPHOGLOSSUM (LOMARIOPSIDACEAE) EN COSTA RICA
}

\author{
Alexánder Fco. Rojas Alvarado \\ Jardín Botánico Lankester, Universidad de Costa Rica, apdo. 1031-7050, Cartago, Costa Rica. \\ afrojasa@yahoo.com
}

\begin{abstract}
Aвstract. The new species Elaphoglossum lenticulatum, sect. Elaphoglossum subsect. Pachyglossa, is described here. This species is segregated from E. proximum (J. Bommer) H. Christ because of entire (vs. ciliate), persistent (vs. deciduous) and bigger rhizome scales, lanceolate (vs. lanceolate-oblong) blade, and less dense and bigger blade scales.
\end{abstract}

Resumen. Se describe la nueva especie Elaphoglossum lenticulatum de la sección Elaphoglossum subsecc. Pachyglossa. Se diferencia de E. proximum (J. Bommer) H. Christ por escamas del rizoma enteras (vs. ciliadas), persistentes (vs. deciduas) y más grandes, lámina foliar lanceolada (vs. lanceolado-oblonga) y escamas de la lámina menos densas y más grandes.

Palabras clave / Kew words: Elaphoglossum, Lomariopsidaceae, Pteridophyta, Costa Rica.

Mickel (1992) describe 33 especies nuevas de Elaphoglossum de Mesoamérica, de las cuales 13 especies pertenecen a la secc. Elaphoglossum subsecc. Pachyglossa y 12 están presentes en Costa Rica. Rojas (1996a) refiere una especie costarricense de Elaphoglossum secc. Elaphoglossum. Rojas (1996b) describe 12 especie nuevas de Elaphoglossum de Costa Rica y Panamá; de ellas, tres son de la secc. Elaphoglossum y todas están presentes en Costa Rica. Rojas (1997) publica 14 especies nuevas de Elaphoglossum de Mesoamérica; siete de ellas de la secc. Elaphoglossum y sólo E. panamense presente en Costa Rica. Posteriormente, Rojas (2002) comunica cuatro registros nuevos de la secc. Elaphoglossum de Costa Rica, y describe (Rojas 2003) 12 especies y registra dos especies más de la subsecc. Pachyglossa de México y Centroamérica, de las cuales cinco especies nuevas y los dos nuevos registros están presentes en Costa Rica. En el presente trabajo se describe una especie nueva más.

Se utilizaron distintos tratamientos taxonómicos (Mickel 1991, 1995, Mickel \& Beitel 1988, Proctor 1985, 1989) y artículos sobre Elaphoglossum, que incluyen especies de Costa Rica (Mickel 1992, Rojas 1996a, 1996b, 1997, 2002, 2003), y se revisaron las colecciones del Herbario Nacional de Costa Rica (CR) y del Instituto Nacional de Biodiversidad (INB).

\section{Elaphoglossum lenticulatum A. Rojas, sp. nova}

TIPO: COSTA RICA. Guanacaste: Liberia, Parque Nacional Guanacaste, Cuenca del Río Tempisque, Volcán Cacao, entre la ramificación del sendero a la
Estación Maritza y la punta del cerro, $10^{\circ} 56^{\prime} 08^{\prime} \mathrm{N}$, 85'27’10”'W, 1300-1600 m, 5 febr 1998, A. Rojas \& A. Soto 4266 (Holotipo: CR, isotipos: INB, MO). Fig. 1.

Species nova haec a Elaphoglosso mitorrhizo Mickel similis, sed rhizomatis squamis maioribus bicoloribusque, laminae squamis ovatis maioribusque dignoscenda.

Epífitas; rizoma de 1-2 mm de diámetro, largamente reptante; escamas del rizoma de 1.5-2.5 (-3.0) x 1-2 $\mathrm{mm}$, ovadas, bicoloras, pardo-amarillentas con el centro y a veces el ápice negruzcos, lustrosas, adpresas, con margen entero; frondas de 7-21 cm, distantes 20-35 mm entre sí; filopodios de 4-8 mm de largo; estípite de 1/32/3 del tamaño de la fronda; escamas del estípite de 1-3 x ca. $1 \mathrm{~mm}$, ovadas a ovado-lanceoladas, bicoloras a concoloras, pardo-amarillentas, adpresas a ligeramente patentes, con margen entero; lámina de 5.5-12 x 2.5$3.5 \mathrm{~cm}$, lanceolada, cartácea, base anchamente cuneada terminando atenuadamente, ápice agudo a acuminado; escamas de la costa de 1-2 x 0.5-1 mm, ovadas, pardas, con margen ciliado; escamas de la lámina de 0.5-1 x 0.3-0.6 mm, ovadas a deltado-lanceoladas, pardas a pardo-oscuras, medianamente densas, con margen dentado a corto-ciliado, ciliado en la base; hidátodos ausentes; frondas fértiles de 11.0-22.5 cm; estípite de 2/5-3/4 del tamaño de la fronda; lámina fértil de 5-7 x 1.6-1.8 cm, lanceolada a angostamente lanceolada, base anchamente cuneada, ápice acuminado; escamas de la lámina presentes sólo en el estípite y la costa, similares a las de la fronda estéril; escamas interesporangiales ausentes. 
Distribución. Conocida sólo de la Cordillera de Guanacaste y la Cordillera de Tilarán, Costa Rica, 1200-2000 m. Especie endémica.

Paratipos: COSTA RICA. Alajuela: Valverde Vega, Cordillera Central, Alto Palomo, cercanías de la cascada y punta del cerro, $10^{\circ} 10^{\prime} 50^{\prime \prime} \mathrm{N}, 84^{\circ} 18^{\prime} 42^{\prime \prime} \mathrm{W}$, 1800-2000 m, 9 mayo 1995, A. Rojas et al. 1816 (CR, INB, MO); San Ramón, Reserva Biológica Monteverde, Cordillera de Tilarán, Estación Aleman's, $10^{\circ} 22^{\prime} 00^{\prime \prime N}, 84^{\circ} 48^{\prime} 00^{\prime \prime W}, 1200-1500$ m, 20 mayo 1995, A. Rojas 1876 (CR, INB, MO). Guanacaste: Fila del Volcán Cacao, 1400-1520 m, 22 sept 1986, I. Chacón \& A. Chacón 2291 (CR); Parque Nacional Guanacaste, Cordillera de Guanacaste, Estación Cacao, $10^{\circ} 55^{\prime} 45^{\prime} \mathrm{N}, 85^{\circ} 28^{\prime} 15^{\prime \prime} \mathrm{W}, 1100-1500 \mathrm{~m}, 5$ sept 1996 , A. Rojas \& M. Mata 3065 (CR, INB, MO).

Esta especie se parece a E. mitorrhizum Mickel por la forma y el tamaño de la lámina, pero se diferencia por las escamas del rizoma más grandes (1.5-3.0 x 1-2 mm vs. 0.5-1.0 x 0.3-0.8 mm), bicoloras (vs. concoloras), pardo-amarillentas con el centro oscuro (vs. pardo-oscuras a negruzcas) y margen entero a ondulado (vs. ciliado) y las escamas de la lámina más grandes (0.5-1 x 0.3-0.6 mm vs. 0.2-0.5 x 0.2-0.3 $\mathrm{mm}$ ), medianamente densas (vs. dispersas) y con el margen dentado (vs. ciliado). También se parece a $E$. proximum (J. Bommer) H. Christ por las escamas del rizoma densas y escamas de la lámina pardo-oscuras a negruzcas, pero difiere por las escamas del rizoma enteras (vs. ciliadas), persistentes (vs. deciduas) y más grandes (1.5-3.0 x 1-2 mm vs 0.7-1.5 x 0.5-1.0 mm), lámina lanceolada (vs. lanceolado-oblonga) y escamas de la lámina menos densas y con margen entero a corto y dispersamente ciliado (vs. margen densa y largamente ciliado) (Figs. 1 y 2, Cuadro 1).

Etimología. El epíteto lenticulatum (con aspecto de lenteja) se refiere a las escamas de la lámina grandes y pardo-oscuras, que se asemejan a pecas.

Agradecimientos. A Carlos O. Morales por las correcciones del latín, a los herbarios del Museo Nacional de Costa Rica (CR) y del Instituto Nacional de Biodiversidad (INB), por permitirme utilizar sus colecciones, y a los revisores anónimos del manuscrito por sus comentarios.

\section{LiTERATURA CITADA}

Mickel, J.T. 1992. New species of the fern genus Elaphoglossum from Mesoamerica. Novon 2: 368382.

Mickel, J.T. 1991. Elaphoglossum. In: Tryon, R.M. \& R.G. Stolze (eds.). Pteridophyta of Peru. Part IV. 17. Dryopteridaceae. Fieldiana, Bot. n.s. 27: 111-166.

Mickel, J.T. 1995. Elaphoglossum. In: Moran, R.C. \& R. Riba (eds.). Flora Mesoamericana. Vol. 1. Psilotaceae a Salviniaceae. Univ. Nac. Autónoma de México. México D. F. p. 250-283.

Proctor, G.R. 1985. Ferns of Jamaica, a guide to the Pteridophytes. British Museum. p. 486-518.

Proctor, G.R. 1989. Ferns of Puerto Rico and the Virgin Islands. Mem. New York Bot. Gard. 53: 169-175.

Rojas, A.F. 1996a . Aportes a la Flora Pteridophyta Costarricense. I. Informes. Brenesia 45-46: 1-6.

Rojas, A.F. 1996b. Twelve New Species of Elaphoglossum from Costa Rica and Panama. Brenesia 45-46: 7-26.

Rojas, A.F. 1997. Fourteen New Species of Elaphoglossum (Elaphoglossaceae) from Mesoamerica. Brenesia 47-48: 1-16.

Rojas, A.F. 2002. New species, new combinations and new distributions in neotropical species of Elaphoglossum (Lomariopsidaceae). Rev. Biol. Trop. 50: 966-1006.

Rojas, A.F. 2003. New taxa in the Elaphoglossum sect. Elaphoglossum subsec. Pachyglossa (Lomariopsidaceae) from Mexico and Central America. Rev. Biol. Trop. 51: 1-32. 
Cuadro 1. Comparación de características morfológicas entre Elaphoglossum lenticulatum, E. mitorhizum у E. proximum.

\begin{tabular}{|l|l|l|l|}
\hline Característica & E. lenticulatum & E. mitorhizum & E. proximum \\
\hline $\begin{array}{l}\text { Largo de las escamas del } \\
\text { rizoma (mm) }\end{array}$ & $1.5-3.0$ & $0.5-1.0$ & $0.7-1.5$ \\
\hline $\begin{array}{l}\text { Ancho de las escamas } \\
\text { del rizoma (mm) }\end{array}$ & $1-2$ & $0.3-0.8$ & $0.5-1$ \\
\hline $\begin{array}{l}\text { Color de las escamas del } \\
\text { rizoma }\end{array}$ & $\begin{array}{l}\text { bicolor, pardo- } \\
\text { amarillento con el centro } \\
\text { oscuro }\end{array}$ & $\begin{array}{l}\text { concolor, pardo-oscuro a } \\
\text { negruzco }\end{array}$ & $\begin{array}{l}\text { concolor, pardo a pardo- } \\
\text { oscuro }\end{array}$ \\
\hline $\begin{array}{l}\text { Margen de las escamas } \\
\text { del rizoma }\end{array}$ & entero a ondulado & ciliado & ciliado \\
\hline $\begin{array}{l}\text { Densidad de las escamas } \\
\text { del rizoma }\end{array}$ & densas & dispersas & densas \\
\hline $\begin{array}{l}\text { Forma de la lámina } \\
\text { foliar }\end{array}$ & lanceolada & lanceolada & lanceolado-oblonga \\
\hline $\begin{array}{l}\text { Largo de las escamas de } \\
\text { la lámina (mm) }\end{array}$ & $0.5-1$ & $0.2-0.5$ & $0.5-1$ \\
\hline $\begin{array}{l}\text { Ancho de las escamas de } \\
\text { la lámina (mm) }\end{array}$ & $0.3-0.6$ & $0.2-0.3$ & $0.3-0.8$ \\
\hline $\begin{array}{l}\text { Margen de las escamas } \\
\text { de la lámina }\end{array}$ & $\begin{array}{l}\text { entero a dentado, ciliado } \\
\text { en la base }\end{array}$ & ciliado & ciliado \\
\hline $\begin{array}{l}\text { Densidad de las escamas } \\
\text { de la lámina }\end{array}$ & medianamente densas & dispersas & densas \\
\hline
\end{tabular}




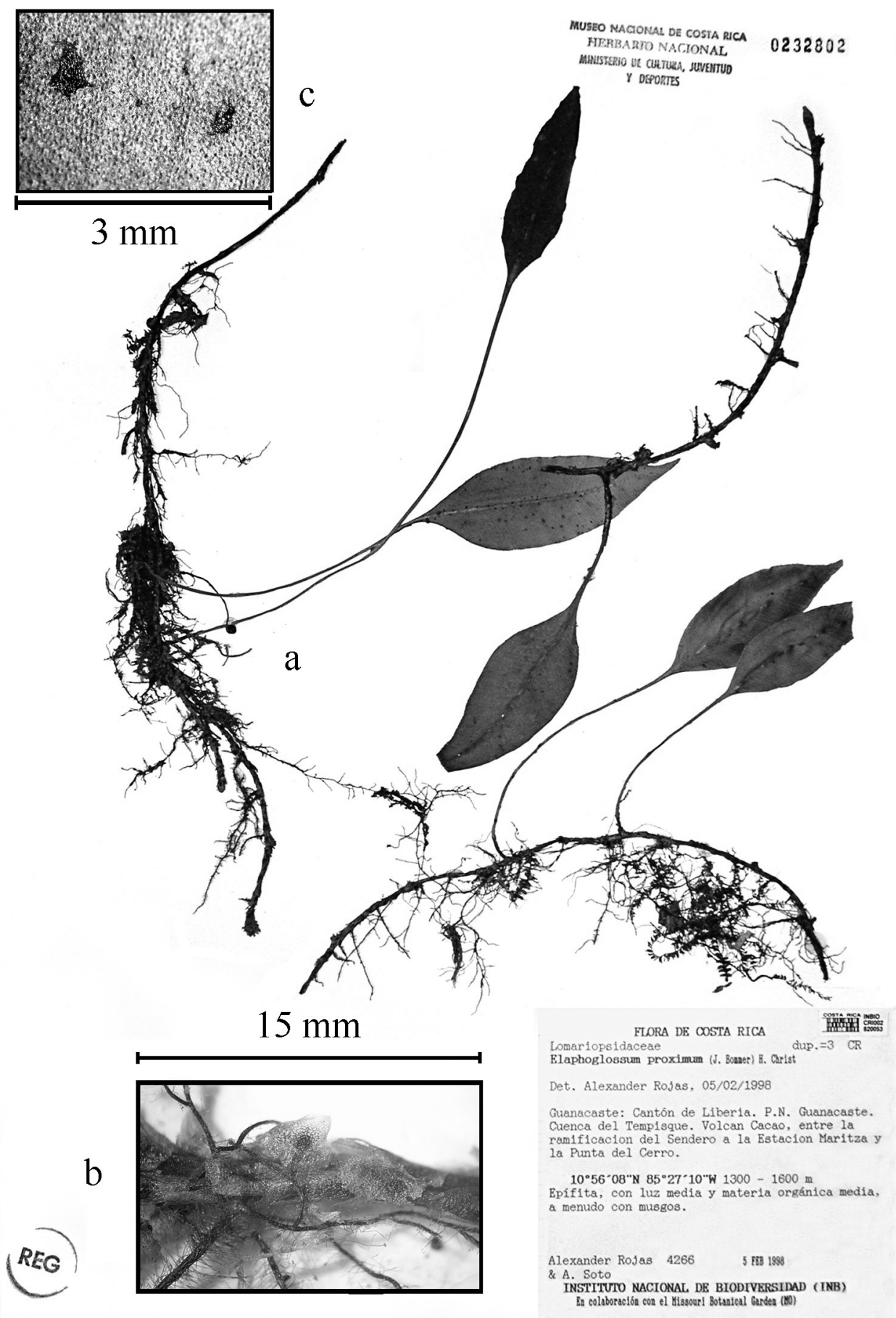

Fig. 1. Tipo de Elaphoglossum lenticulatum (A. Rojas \& A. Soto 4266, CR): a. Hábito. b. Detalle del rizoma. c. Detalle de la lámina foliar. 


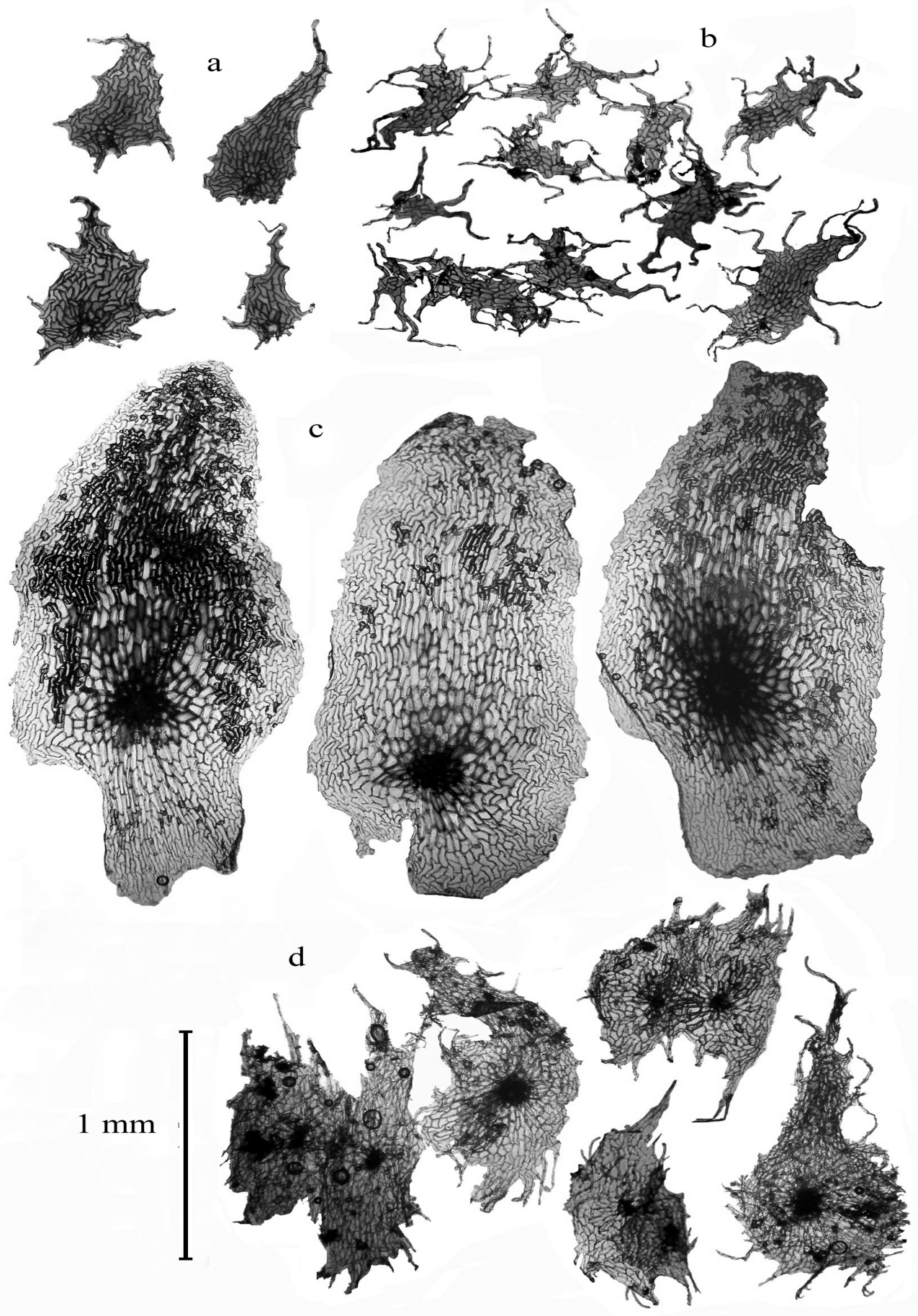

Fig. 2. Elaphoglossum lenticulatum (A. Rojas \& A. Soto 4266, CR): a. Escamas de la lámina. c. Escamas del rizoma. E. proximum (A. Rojas 3392, CR): b. Escamas de la lámina. d. Escamas del rizoma. 
